\title{
EL CARÁCTER INTERACTORAL EN LA EDUCACIÓN SUPERIOR CON ENFOQUE INTERCULTURAL EN MÉXICO
}

\author{
The Nature of Interactions in Mexican Higher Education with an Intercultural Approach
}

\author{
Angélica Rojas-Cortés \\ Erica González-Apodaca
}

Resumen: En México la educación superior intercultural ha estado signada por la política oficial. La Coordinación General de Educación Intercultural y Bilingüe (CGEIB) ha creado las llamadas universidades interculturales; sin embargo, anteriores y paralelas a éstas operan otras instituciones con enfoque intercultural fuera de esta política de Estado, con sentidos diversos en su carácter intercultural. A partir de una revisión de trabajos analíticos y descriptivos, de investigación y divulgación, en el presente artículo se explora el carácter interactoral de diversas instituciones de educación superior intercultural en México y se identifican algunos retos que emanan de esta dimensión.

Palabras clave: interculturalidad, educación superior, pueblos indígenas, investigación social, método interactoral.

Abstract: Mexico is different from other countries in that intercultural higher learning has become politically sanctioned. The General Coordinator of Intercultural and Bilingual Education has created so-called Intercultural Universities, however prior and parallel to these, other institutions with an intercultural focus have emerged outside of national public educational system. Based on a review of scientific bibliography, both analytic and descriptive, this article tries to explore the quality of the intercultural exchanges between actors at some of these educational centers in Mexico.

Keywords: interculturality, higher education, indigenous people, social investigation, interaction methodologies.

\footnotetext{
Angélica Rojas Cortés, doctorado en Ciencias Sociales con especialidad en Antropología Social por el CIESAS Occidente, México. Investigadora en CIESAS Pacífico Sur como parte del programa Cátedras CONACYT, México. Temas de especialización: educación, interculturalidad y etnicidad. Correo electrónico: angelicaroc@hotmail.com.

Erica González Apodaca, doctorado en Ciencias Antropológicas por la Universidad Nacional Autónoma de México. Profesora-investigadora en el CIESAS Pacífico Sur, México. Temas de especialización: antropología e historia de la educación, escuela y etnicidad y educación intercultural. Correo electrónico: erika.g.apodaca@gmail.com.
}

Enviado a dictamen: 19 de mayo de 2015.

Aprobación: 13 de octubre de 2015.

Revisiones: 1. 


\section{Introducción}

$\amalg$ n las últimas décadas del siglo XX, en el marco de las políticas de reconocimiento a la diversidad, documentos emanados de reuniones internacionales en el ámbito de la educación superior incorporaron el eje de la diversidad cultural. En 1998, la Declaración Mundial sobre Educación Superior en el siglo XXI: Visión y Acción de la UNESCO estableció un enfoque de acción afirmativa en materia de acceso de "grupos minoritarios" y culturalmente diversos a las instituciones de educación superior, en la que se indicaba que:

Se debe facilitar activamente el acceso a la educación superior de los miembros de algunos grupos específicos, como los pueblos indígenas, las minorías culturales y lingüísticas, de grupos desfavorecidos, de pueblos que viven en situación de ocupación y personas que sufren discapacidades [...] Una asistencia material especial y soluciones educativas pueden contribuir a superar los obstáculos con que tropiezan esos grupos, tanto para tener acceso a la educación superior como para llevar a cabo estudios en ese nivel (artículo 3, D).

En el documento también se subrayó la necesidad de colaboración entre los responsables de las políticas nacionales e institucionales, el personal docente y administrativo, los estudiantes, el mundo laboral, las organizaciones no gubernamentales y los grupos comunitarios, y se hacían recomendaciones diversas en torno a los currículos nacionales y otros temas. El Primer (2002) y Segundo (2003) Encuentros Regionales de Educación Superior para los Pueblos Indígenas de América Latina, reportados por Padilla (2008), ahondaron en las líneas esbozadas. Las declaratorias internacionales favorecieron, según algunos, la institucionalización de la política intercultural en el nivel de educación superior en México (Bello, 2011).

Sin embargo el tema rebasa el acceso de "grupos minoritarios" a la educación superior, y se vincula con el reconocimiento del derecho a la diversidad cultural y de los derechos educativos diferenciados de los pueblos indígenas. Entre estos últimos figura el derecho a acceder a una educación con pertinencia cultural y lingüística en todos los niveles y a participar en su definición, implementación y gestión, derechos enunciados en el Convenio 169 dela Organización Internacional del Trabajo (OIT) y en documentos jurídicos internacionales. Las recomendaciones y derechos reconocidos han permeado el debate en espacios políticos, civiles y académicos, y favorecido la creación y difusión de iniciativas de educación superior con enfoque intercultural en América Latina y en México. Como muestra el trabajo de Mato (2008), las organizaciones indígenas y afrodescendientes han asumido un papel protagónico en los contextos poscoloniales latinoamericanos; a diferencia de las iniciativas promovidas por los Gobiernos, las propuestas enarboladas por el movimiento social e indígena se sustentan en la reivindicación positiva de la identidad y la cultura "propias", la crítica a las formas hegemónicas de conocimiento y saber, la legitimidad de saberes "otros" vinculados con la praxis cultural y política de los actores, y la defensa del territorio y los recursos colectivos (Gasché, 2008; López Hurtado, 2010; Walsh, 2010; Bertely, 2013). Tales significados no son foco de interés en las agendas gubernamentales de educación intercultural.

Para Mato, el carácter heterogéneo de la educación superior intercultural en América Latina tiene que ver con: a) la diversidad misma de pueblos y comunidades donde están proyectadas, b) la diversidad de países donde han sido concebidas y c) la diversidad de actores que impulsan estos proyectos (Mato, 2008). En México, según Dietz, la educación superior intercultural se conforma como un subsistema universitario que promociona una formación académica culturalmente pertinente a estudiantes diferenciados en términos étnicos, lingüísticos o culturales; lo cual, señala, luego de casi un siglo de políticas indigenistas y neoindigenistas de Estado, no impacta en la universidad pública, como el centro donde se construye y difunde el "conocimiento universal, occidental y colonial” (Dietz, 2010).

Ubicándonos en estos debates, el objetivo del presente artículo es explorar el carácter interactoral heterogéneo de la educación superior intercultural en 
México e identificar algunos retos que se desprenden del mismo, mediante una revisión de productos de investigación y trabajos de divulgación realizados sobre el tema entre 2008 y 2015, periodo en el que se encontró mayor producción. Concebimos la educación superior intercultural como un campo social disputado, compuesto por redes socioeducativas diversas, dinámicas y en tensión. Exploramos su carácter interactoral en dos ámbitos que atraviesan los trabajos: el primero se centra en las "concepciones de interculturalidad" presentes en las instituciones de educación superior -en sus actores y en los discursos instituidos e instituyentes - ; y el segundo en la "génesis negociada" de las instituciones de educación superior reportadas en la producción que, al estar inmersas en redes socioeducativas heterogéneas e interactuar con las dinámicas sociales y políticas regionales, adquieren rasgos distintivos y cuestionan la viabilidad de modelos "replicables".

En el estado del conocimiento en multiculturalismo y educación en la década 2002-201l (Bertely, Dietz y Díaz, 2013), la educación superior intercultural es un tema que atraviesa varias áreas de investigación. Se aborda, al menos, desde: a) las políticas públicas oficiales, en contraste con iniciativas no tuteladas por el Estado (Velasco y Jablonska, 2013); b) currículos $\mathrm{y}$ acciones afirmativas en universidades y posgrados convencionales y escuelas normales (Mateos, Mendoza y Dietz 2013); c) iniciativas etnogenéticas (González y Rojas, 2013); d) el horizonte del pensamiento decolonial (Medina y Baronnet, 2013); e) la formación de docentes en y para la educación intercultural (Von Groll, Keyser y Silva, 2013), y f) el locus de las universidades interculturales de la CGEIB (Dietz y Mateos, 2013). También es categorizada de acuerdo con el tipo de vinculación establecida con el Estado y con los alcances de una enunciación autónoma o semiautónoma (Bertely, 2011) respecto de sus instituciones y recursos.

Estos estudios son punto de referencia para este artículo, que consta de cinco apartados. Se inicia con una descripción de los aspectos metodológicos que orientaron la revisión de los trabajos y sus características centrales. Posteriormente se apunta la utilidad de una concepción política y significante de la interculturalidad como plataforma, para adentrarse en el carácter heterogéneo de la educación superior intercultural y en las disputas por la relación entre poder y sentido que la atraviesan y que ponen en juego "diferentes representaciones colectivas en torno a la equidad social" (Sartorello, 2009: 87). En el tercer apartado se describe la producción, enfocando las perspectivas de interculturalidad que se destacan en los trabajos y que emanan de los discursos institucionales, de los actores o de los mismos autores "en tanto actores" académicos en el campo. En el cuarto apartado se recupera la génesis y conformación heterogénea de las instituciones documentadas y las posiciones a menudo confrontadas de sus actores, partícipes de relaciones de poder. Finalmente, el texto se cierra subrayándose los retos que se desprenden del análisis de los aspectos anteriores en este campo de investigación y de intervención social.

\section{Aspectos metodológicos}

La selección de las fuentes se orientó hacia trabajos de investigación académica o productos de divulgación publicados en México; por razones de espacio quedaron fuera de esta primera revisión los trabajos publicados en el extranjero y los documentos normativos, programáticos y de políticas educativas. Los productos incluyen dos tipos de fuentes: a) investigaciones concluidas que tienen un objetivo analítico y dialogan con un andamiaje teórico, y b) trabajos de divulgación de experiencias educativas interculturales que documentan los deberes y haceres de las instituciones. Se revisaron treinta y cuatro textos en total: dos libros, diecisiete capítulos de libros, tres ponencias, tres tesis de grado y posgrado y nueve artículos de revistas académicas especializadas.

De igual forma que los estudios interculturales, el campo de la educación superior intercultural en México se perfila como multidisciplinario; en él confluyen principalmente la antropología, la sociología, la ciencia política, la pedagogía y las ciencias de la educación. Empero, los trabajos de divulgación no necesariamente 
se inscriben en una tradición disciplinaria específica y buena parte de la producción revisada no explicita sus marcos teórico-metodológicos. La mayoría - quincese refiere a las universidades interculturales de la Coordinación General de Educación Intercultural y Bilingüe de la Secretaría de Educación Pública (CGEIBSEP) (Ávila, 2009; Bailleres, 2010; Dietz, 2008 y 2009; Fábregas, 2008 y 2009; Guerra, 2008; Guerra y Meza, 2009; Guitart y Rivas, 2008; González, 2007; Hernández, 2012; Hernández y Lemus, 2013; López, 2013; Silva, 2009). Siete trabajos tratan sobre ellas en lo general y con fines analíticos (Bello, 2011; Bertely, 2011; Dietz, 2014; Lehman, 2015; Ortelly y Sartorello 201l; Padilla, 2008; Sandoval y Meza 2010; Vivar, 2014). En contraste, sólo siete estudios se refieren a universidades o centros de educación superior que funcionan fuera de esta estructura (Alonso et al., 2014; Cantú et al., 2006; Estrada et al., 2006; Estrada, 2008; Flores, 2009; López Rangel, 2009, s.f. y 2013; Maldonado, 2014).

Un dato significativo es que una parte de los trabajos se produce en el seno de las propias universidades a las que pertenecen sus autores, y algunos son autorreferenciales. ${ }^{1}$ Los espacios de difusión son revistas académicas nacionales y memorias de congresos, en particular del Consejo Mexicano de Ciencias Sociales, el Consejo Mexicano de Investigación Educativa y los Encuentros Regionales sobre Educación Superior Intercultural organizados por el Instituto Internación para la Educación Superior en América Latina y el Caribe, y la CGEIB-SEP. En estos foros se aprecia la participación incipiente de egresados de las instituciones de educación superior.

\section{Interculturalidad y educación superior intercultural en México}

La institucionalización de la educación superior intercultural en 2001, con la creación de la CGEIBSEP, instrumentó la creación de las universidades interculturales en contextos de población indígena ${ }^{2}$ que, si bien operan bajo un modelo general y lineamientos comunes, son heterogéneas en su origen, la composición del alumnado y del profesorado, sus articulaciones regionales y estatales y sus condiciones de operación. La institución y la cultura escolar en cada caso no son un mero reflejo de las políticas, antes bien se construyen en interacción permanente con el contexto social, político y sociocultural, y su devenir histórico.

Sin embargo, podemos afirmar que la interculturalización de la educación superior es anterior a la creación de las universidades interculturales, ${ }^{3}$ aunque las iniciativas suscriban otras denominaciones. Un primer ámbito es la formación de profesores, vinculada con la educación indígena, cuyo devenir se actualizó con la incorporación del enfoque intercultural a la educación básica en 1992, lo que llevó a la apertura de escuelas normales interculturales y a propuestas de transversalización curricular en la formación de docentes indígenas, así como al surgimiento de iniciativas comunitarias y etnopolíticas autonómicas que han impulsado novedosos espacios de formación docente intercultural. ${ }^{4}$

En un segundo ámbito, desde finales de los años ochenta se gestaron iniciativas de educación superior intercultural en contextos de composición étnica o multiétnica. Enunciadas como campesinas, comunitarias o interculturales, desarrollaron propuestas de profesionalización a partir de criterios de pertinencia cultural y política, y de educación de calidad. En este ámbito — también heterogéneo-, las instituciones de educación superior tienen posiciones de mayor o menor autonomía frente a la SEP y los recursos educativos del Estado; si bien algunas fueron absorbidas posteriormente por la CGEIB, otras subsisten bajo esquemas diversos de gestión, certificación y financiamiento, generalmente en la estructura educativa del Estado y participando de redes interactorales diversas. Una de las más antiguas, que data de 1989, es la Licenciatura en Desarrollo Rural del Centro de Educación Superior para el Desarrollo Rural (CESDER A.C.), en el municipio de Zautla, en la sierra norte de Puebla. ${ }^{5}$ Vinculada a esta experiencia surgió en 1999 la Universidad Campesina Indígena en Red (UCIRed).

¿Cómo explicar entonces un campo socioeducativo, político y académico que semantiza lo intercultural desde una gama tan heterogénea de posiciones, perspectivas y demandas? 
Sin pretender agotar la respuesta, subrayamos una doble dimensión del concepto como andamiaje para orientar la revisión. En primer lugar partimos de una dimensión política. Pérez Ruiz (2009) aclara que con el concepto de interculturalidad se suele aludir simultáneamente tanto a un discurso prescriptivo de valores éticos de la convivencia intercultural, como a un hecho social de interacción entre "poblaciones con diferencias étnicas, identitarias, culturales, jurídicas y/o religiosas" (Pérez, 2009: 252). El concepto actual pone de relieve la puesta en práctica de distintos -y contrapuestos - proyectos políticos construidos por los actores partícipes de esas relaciones, que se caracterizan por ser relaciones de desigualdad, en contextos de diversidad cultural. Inicialmente puede distinguirse, nos dice la autora, una vertiente multicultural fundada en una "filosofía de la tolerancia" de una vertiente latinoamericana, "asociada con los movimientos indígenas, descolonizadores y de liberación nacional", que le imprime al concepto "contenidos específicos, relacionados con los proyectos políticos con los que se vincula" (Pérez, 2009: 255-256).

Desde esta perspectiva, la interculturalidad no se resuelve en el terreno ético-pedagógico, sino que tiene una connotación política y una naturaleza inherentemente conflictiva (Gasché, 2008); sin embargo, la semantización diversa de lo intercultural es más compleja. Pérez Ruiz señala, por ejemplo, las diferencias que emergen "cuando para unos la interculturalidad se asocia sólo con la lucha por el reconocimiento o cuando, por el contrario, se articula con proyectos de transformación social, que incluyen la refundación de las naciones" (Pérez, 2009: 256). En su dimensión política, involucra propuestas y horizontes societales diversos que se gestan en relaciones de poder y desigualdad.

Como segunda dimensión o vértice de lo intercultural, resaltamos su carácter de discurso significante (González Apodaca, 2009), que implica la imposibilidad de definirla en abstracto; antes bien, como también se desprende de la dimensión política, sus sentidos varían según los contextos de definición y los actores implicados, así como según las posiciones que ocupan y desde las cuales negocian su contenido. Coincidimos con González Apodaca en que: "concebir la interculturalidad como significante implica profundizar en los planteamientos políticos y filosóficos que sustentan las diferentes propuestas educativas interculturales y bilingües que se generan desde distintos actores sociales" (González Apodaca, 2009: 87), los cuales, al situarse en campos sociales o arenas políticas, implican una tensión entre diversas formas de concebir un proyecto societal, sus actores y sus relaciones intra e interculturales, o lo que este autor denomina las "filosofías políticas" (González Apodaca, 2009: 87) distintivas que inspiran las propuestas de educación superior intercultural y sus marcos políticojurídicos, epistemológicos y pedagógicos.

Finalmente, asumimos el análisis de Dietz y Mateos (2011) en el sentido de que el campo normativo-político y de investigación de la interculturalidad y la educación intercultural en México es heredero de un sesgo indigenista y neoindigenista de las políticas oficiales posrevolucionarias dirigidas a integrar a la población indígena a la sociedad nacional (Dietz y Mateos, 2011). Uno de los retos es avanzar hacia perspectivas interculturales que consideren la "diversidad" como una categoría no circunscrita a la diferencia étnica.

\section{Nociones de interculturalidad en el campo de investigación}

Una pregunta recurrente en los trabajos remite a las concepciones de interculturalidad y de educación intercultural que operan en las instituciones y que informan el currículo y las prácticas escolares. Metodológicamente, los trabajos indagan en los "discursos actorales" tanto de autoridades educativas, como de profesores. ${ }^{6}$ Sandoval y Guerra (2007) analizaron las perspectivas de los rectores de las universidades interculturales y encontraron que identifican la educación intercultural con el desarrollo de currículos flexibles y de sumatorias que incorporen saberes de "todas las culturas". La polisemia del concepto los lleva a interrogarse sobre la interculturalidad como un hecho social o un objetivo por alcanzar. 
Se señala que los "discursos institucionales" de las universidades interculturales, analizados en documentos y prácticas escolares, suelen reflejar la prevalencia de una noción indigenista sobre la interculturalidad que imprime a las prácticas un carácter homogenizador y una visión ahistórica de la cultura. De igual manera, se identifica el empuje de una perspectiva empresarial de la interculturalidad, que subraya la incidencia de las reformas neoliberales en las políticas de educación superior para que respondan a las lógicas del mercado global. En este sentido, Padilla (2008) y Llanes (2008) argumentan que los valores de competencia, productividad y delimitación de responsabilidades configuran un concepto de "interculturalidad funcional" en las universidades interculturales, que supone que los sujetos indígenas deben profesionalizarse bajo criterios desarrollistas que obedecen a intereses empresariales. El sesgo ideológico consiste en que la diversidad se valora en la medida en que permite el ejercicio de los derechos individuales, basados en principios universales y funcionales a las políticas neoliberales contemporáneas. En esta misma línea se ubican estudios críticos del carácter intercultural de estas universidades como parte de una tendencia a "politizar la cultura y despolitizar la economía" (Aguilar, 2011, citando a Díaz Polanco) y como una forma contemporánea del multiculturalismo aliado con el capitalismo global que tiende a generar nuevas formas de colonialismo interno (Guerra, 2008; Sandoval y Guerra, 2008).

Otro grupo de trabajos influidos por una perspectiva latinoamericanista recurre a una noción de interculturalidad como proyecto descolonizador, fundada en el pensamiento de Aníbal Quijano, Boaventura de Souza y Catherine Walsh. Ésta se basa en el reconocimiento de epistemologías y saberes "otros", y cuestiona la universalidad de conocimientos hegemónicos, en aras de construir unas ciencias sociales críticas de la racionalidad occidental. Los trabajos de Aguilar (2011), Hernández (2012), Llanes (2008) y Vázquez (2014) sobre las universidades interculturales consideran la educación intercultural como un proyecto político-educativo que reflexiona sobre la colonialidad del poder, del conocimiento y de sus habitus institucionales, y asumen posiciones críticas sobre el carácter uninacional y monocultural de la educación superior en general y de la educación superior intercultural institucionalizada en México.

La mayoría de los trabajos, tanto los que estudian las universidades interculturales como las investigaciones sobre experiencias de educación superior intercultural no vinculadas orgánicamente a la CGEIB, desarrollan un concepto de interculturalidad más cercano a la antropología y la sociología políticas. La interculturalidad conflictiva o crítica (Gasché, 2008; Bertely, 2007) forma parte de arenas políticas donde se debaten proyectos diversos e intereses confrontados (González Apodaca, 2008), y se reconocen las asimetrías sociales y de poder en juego. En general, desde andamiajes conceptuales diversos, se ubica el conflicto como constitutivo de las relaciones interculturales y se preguntan por la pertinencia cultural y política de las propuestas (Bertely, 2011). Se subraya también, desde esta perspectiva, la invisibilización de la "desigualdad", dimensión ausente en la perspectiva intercultural de las universidades interculturales (Lehman, 2015; López, 2013; Vivar, 2014).

Por su parte, las investigaciones que suscriben la noción de la "interculturalidad crítica" (Walsh, 2010) tienen carácter evaluativo y hasta cierto punto prescriptivo; en general parten del supuesto de que ésta tendría que ser la base política e ideológica fundante de las experiencias y proyectos de educación superior intercultural en el país.

En otro ángulo, destacan las relaciones conceptuales que se tejen entre los conceptos de "interculturalidad" y de "comunidad", abordadas por Navarro (2012) en su artículo sobre la Universidad Intercultural del Estado de Tabasco (UIET). El autor reflexiona sobre el papel de la UIET a nivel comunitario, y concluye que la comunidad representa un espacio de intervención del proceso de desarrollo regional que el Estado deja en manos de las universidades interculturales, y no una unidad de análisis sobre la que hay que reflexionar en tanto copartícipe del mismo proceso de interculturalización. Argumenta que, en esta lógica, el proyecto social de 
la educación superior intercultural y su incidencia en las comunidades se reduce a un asunto de interés público circunscrito al ámbito universitario, y no a una vinculación efectiva con la comunidad sociohistórica.

Por su parte, Lehman (2015) desarrolla un análisis en el que contrasta las perspectivas de interculturalidad presentes en el discurso de los rectores de cinco universidades interculturales, en los documentos prescriptivos de las instituciones y en las percepciones del profesorado. Distingue dos posturas: en un caso, la interculturalidad no se circunscribe a la población indígena, sino que persigue una relación de equidad entre conocimientos y culturas diferentes, como un proyecto ciudadano orientado a la igualdad en la diversidad. En los otros cuatro casos, por el contrario, lo intercultural se asocia con la concientización de los miembros de la cultura dominante sobre el valor de otras culturas, y con el aumento de la competencia de los grupos culturales subalternos - los indígenas-en la cultura y la lengua dominantes, un proceso que hipotéticamente tiene lugar en un contexto de respeto que propicia el diálogo entre iguales. El autor reporta que, para la mayoría de los profesores de las universidades interculturales entrevistados, el objetivo de éstas es formar profesionales que contribuyan al desarrollo del país y sean competentes en los marcos culturales hegemónicos; con excepción del primer caso que muestra, ${ }^{7}$ la cultura comunitaria no parece ser relevante más que como puente para lograr las competencias en la cultura nacional y como forma de legitimación de las políticas compensatorias del Estado. El autor también considera que se sobredimensiona la afirmación positiva de los elementos culturales comunitarios y que se pierde de vista que la política situada tras este discurso es una mera acción afirmativa y no una política de exigibilidad de derechos. Esto pone en duda la supuesta contribución de las universidades interculturales a la superación de las desigualdades socioeconómicas y educativas asociadas con la condición étnica y rural.

Las críticas desarrolladas hacia las universidades interculturales de la SEP iluminan algunos de sus retos. Las implicaciones de ser instituciones construidas "desde arriba", sin una participación sustantiva de los actores locales, plantea el reto de ampliar las formas de vinculación entre las instituciones de educación superior y los actores sociales comunitarios y regionales como ruta para construir la pertinencia social, educativa y política de la educación superior intercultural. Asimismo, la génesis de las universidades interculturales como parte de una política neoindigenista, con prevalencia de concepciones sustancialistas y ahistóricas de la cultura y la identidad, incide en la folclorización y mercantilización de las identidades indígenas contemporáneas y en la estigmatización de los sujetos, imponiendo estereotipos y clasificaciones generadas por el Estado neoliberal contemporáneo y su alianza con el mercado y la empresa privada. Esto impide profundizar en el conocimiento de las prácticas culturales propias e híbridas de los pueblos indígenas contemporáneos y en los conocimientos local-globales implícitos en ellas. Se requiere, por tanto, de incorporar visiones procesuales y constructivistas de la cultura y la identidad que reflejen quiénes son los sujetos indígenas hoy día y las formas diversas en que construyen sus pertenencias múltiples.

De igual manera, las contradicciones entre los objetivos institucionales, los enfoques de los profesores y los intereses de los estudiantes de las universidades interculturales, quienes en muchos casos recurren a éstas como la única opción a la que tienen acceso, evidencian el reto de superar la reificación de lo cultural y la invisibilidad del tema de la desigualdad en ellas, que no suelen problematizar las estructuras de desigualdad basadas en la condición racial, étnica, de clase, de edad o de género como constitutivas de lo intercultural (Bailleres, 2010; Guerra, 2008; Guerra y Meza, 2009; Guerra y Sandoval, 2007; Vivar, 2014). En general se reportan concepciones divergentes y contradictorias de interculturalidad entre los actores universitarios, y distancia entre sus discursos y sus prácticas concretas. Sin embargo, la falta de investigaciones sobre casos no vinculados con la educación superior intercultural oficial aún no permite visibilizar otras tendencias. Un reto del campo de investigación de la educación superior intercultural en México es la elaboración de estudios sobre iniciativas y proyectos instituyentes, cuya ausencia sigue siendo notoria. 
Ante los retos, algunos autores aportan propuestas que tienen como fondo común la transformación crítica de las nociones de interculturalidad. Guerra y Sandoval (2007) proponen subvertir la continuidad del proceso de aculturación en las universidades interculturales mediante una "educación transcultural societal" que visibilice las relaciones asimétricas interculturales y enfatice una dimensión político-cultural en la formación. Dietz (2014) señala que las visiones esencializantes de la identidad indígena constituyen un claro sesgo en las concepciones sobre la diversidad de las instituciones de educación superior intercultural. Subraya la necesidad de emprender un proceso reflexivo que promueva un cambio de enfoque para transitar de una perspectiva indigenista, a una de reconocimiento de la diversidad.

Aguilar (2011) propone cuestionar lo intercultural como adjetivo, que a su juicio remite únicamente a la interculturalización del currículo, y señala la necesidad de reconocer las tensiones e intermediaciones en el juego entre lo instituido y lo instituyente, así como las "identidades en proceso", con miras a establecer lo sustantivo de la interculturalidad. Finalmente, Bertely (2011) señala que el principal reto de la educación superior intercultural - tanto gubernamental como autónoma- es la inclusión de dimensiones como el conflicto, el poder, las desigualdades y la dominación frente a un Estado que no responde a la diversidad cultural de la "nación". En su perspectiva, esto implica ir más allá de políticas compensatorias de acción afirmativa y construir proyectos educativos autonómicos en el nivel.

\section{Génesis y redes interactorales en la educación superior intercultural}

Los trabajos revisados confirman que, como sucede en otros niveles educativos, la educación intercultural en México involucra múltiples interfaces constitutivas de una dimensión interactoral en la que confluyen actores con intereses diversos, "cada uno con sus propias agendas" (Dietz y Mateos, 2011). Estas relaciones configuran redes interactorales complejas, cuyos actores establecen alianzas coyunturales entre sí y disputan sus cuotas de control sobre distintos recursos materiales y simbólicos en juego.

La investigación socio-antropológica e histórica de procesos educativos en contextos interculturales (reseñada, entre otros, en Bertely, 2003; Bertely, Dietz y Díaz, 2013; Rockwell y González, 2012) ha mostrado que la configuración de sus redes socioeducativas no es circunstancial, sino que se inscribe en procesos históricos que explican las relaciones específicas y multidireccionales de los actores locales con las políticas educativas del Estado, las escuelas y el saber escolar (Bertely, 2003). Sin embargo, aunque esta línea de investigación se ha ampliado considerablemente en las últimas dos décadas (Bertely, Dietz y Díaz, 2013), son relativamente escasos los trabajos en el nivel de educación superior. Un pendiente es el desarrollo de investigaciones de larga data que documenten estos procesos en las instituciones de educación superior intercultural en contextos rural-urbanos, comunitarios y migratorios.

Sobre las universidades interculturales oficiales, se enfatiza que su génesis y desarrollo son procesos interactorales heterogéneos y con diversos equilibrios de poder. De acuerdo con Karla Vivar (2014), estas universidades representan un capital político significativo para los gobiernos, que se refleja en sus prácticas de administración y gestión, como el nombramiento de los rectores por los gobernadores de los estados.

Sin embargo la estructura no es uniforme; algunas universidades interculturales fueron independientes en su origen, previo a la creación de la CGEIB, y posteriormente fueron absorbidas por esta instancia como estrategia para solventar sus necesidades de institucionalización. Es el caso de la Universidad Autónoma Indígena de México (UAIM), fundada en diciembre del año 2001 en El Fuerte, Sinaloa, estudiada por Guerra (2008). Esta institución provino del Departamento de Etnología de la Universidad de Occidente en Los Mochis, espacio al que llegaban numerosos aspirantes de origen indígena. El aumento en la demanda llevó a la constitución de la UAIM, una de las primeras instituciones de educación 
superior multiétnicas con estudiantes ch'oles, mames, zoques, kakchikeles, tseltales, tsotsiles, mazahuas, zapotecos, mixtecos, purépechas, nahuas, coras, pimas, rarámuris y yoremes de Sinaloa y Sonora. Posteriormente, problemas políticos internos y limitaciones presupuestales llevaron a un conflicto institucional que provocó la salida del primer rector, ante lo cual se consideró la oferta de incorporarse a la CGEIB-SEP. Su incorporación tuvo lugar en el año 2005 (Guerra, 2008; Guerra y Meza, 2009).

Un caso similar que se gesta al interior de universidades convencionales es la Universidad Veracruzana Intercultural (UVI), que se originó en la línea de investigación en educación multi e intercultural, del Instituto de Investigaciones en Educación de la Universidad Veracruzana (IIE-UV) (Dietz, 2008). Este caso se distingue por el peso de actores académicos que estaban involucrados en proyectos de investigación participativa en contextos interculturales, quienes promovieron seminarios y cursos de especialización y posgrado como espacios de formación para profesionistas provenientes de diferentes contextos indígenas de Veracruz.

De estas iniciativas años más tarde surge la UVI, inicialmente impulsada por académicos formados en el campo de los estudios culturales y que sostenían una concepción constructivista de la cultura. Estos actores se interesaron por desarrollar una propuesta educativa a nivel superior, conforme al contexto pluriétnico estatal, que formara a los alumnos en las competencias necesarias para interactuar en complejos contextos sociopolíticos interculturales. Para llevar a cabo esta empresa tejieron redes con profesionistas, etnolingüistas y activistas indígenas, que no necesariamente compartían la misma visión de interculturalidad, sino que buscaban una educación diferencial que formara parte de un proceso de empoderamiento. Las posturas divergentes implicaron intercambios y negociaciones que tuvieron como resultado la creación de un modelo que conjugaba ambas perspectivas. En estos intercambios se involucraron también actores con conocimiento del contexto, como organizaciones no gubernamentales y movimientos sociales, entre ellos de corte ecologista (Dietz, 2009), por lo que se integró un tejido interactoral que devino en una propuesta de educación intercultural negociada.

Finalmente, Fábregas (2008 y 2009) abordó la construcción de la Universidad Intercultural de Chiapas (UNICH), y en sus análisis destacó que para su conformación, por mandato del gobernador a través de la Secretaría de Educación del estado, se conformó un grupo de trabajo integrado por la Secretaría de Pueblos Indios, el Centro de Lenguas, Artes y Literatura Indígenas de Chiapas, la CGEIB, educadores indígenas, antropólogos y representantes comunitarios. El grupo se disolvió una vez creada la nueva universidad.

Asimismo, la fundación de esta universidad intercultural estuvo precedida por expectativas y enfoques contradictorios, sobre todo entre los intelectuales indígenas y los actores académicos. Los primeros expresaban la expectativa de una educación de nivel superior que fortaleciera la cultura de las comunidades indígenas, mientras que ciertos actores académicos argumentaban que esa propuesta era discriminatoria para los no indígenas y pugnaban porque el presupuesto asignado a la nueva institución fuera utilizado para fortalecer las universidades públicas ya existentes. Los autores afirman que en la génesis de la UNICH se pusieron en juego relaciones interculturales discriminatorias y racistas, como proyección del contexto sociohistórico donde esta institución se inserta (Ortelli y Sartorello, 2011).

En síntesis, estas investigaciones muestran diferentes intencionalidades y lógicas políticas que influyen en la apertura de las instituciones de educación superior en cuestión. Su carácter intercultural político se construye en formas y énfasis distintivos, que corresponden a dinámicas y correlaciones de fuerza regionales. Las diferentes intencionalidades políticas pueden legitimar proyectos y posiciones hegemónicos; sin embargo, están pendientes estudios cualitativos que profundicen en el análisis de la agencia social de los involucrados y en sus prácticas de resistencia, apropiación, negociación o contestación, que semantizan los proyectos de interculturalización de la educación superior en cada caso. También son 
urgentes trabajos que aborden el desarrollo de las Universidades Interculturales de la CGEIB desde los procesos -inacabados - de construcción del Estado pluralista contemporáneo y sus políticas neoliberales, sobre todo en regiones caracterizadas por fuertes tensiones intra e interculturales, despojos territoriales, migraciones trasnacionales, iniciativas de organización social y procesos de desigualdad, exclusión y violencia.

La génesis de la educación superior intercultural en contextos interculturales involucra relaciones de poder, no sólo en relación con la lucha por recursos materiales y humanos, sino en torno a las disputas por los "significados" que se hegemonizan con la institucionalización, y las clasificaciones y heteroclasificaciones dominantes y subalternas. En la península de Yucatán, Genner Llanes (2008) reportó un proceso de "interculturalización fallida" en la gestión de una universidad maya, resaltando las distintas definiciones de "lo maya" que se pusieron en juego. Al documentar las reuniones sostenidas tras el anuncio de creación de la universidad intercultural por el gobernador del estado, Llanes identificó los intereses contrapuestos entre empresarios y sus aliados gubernamentales, que negociaban un perfil de egreso como profesionistas funcionales a la industria turística, y los representantes de las organizaciones mayas, que defendían un proyecto de pertinencia lingüística y cultural. En esta arena, la propuesta devino finalmente en la creación de una universidad no intercultural, con las carreras de Gastronomía y Desarrollo Turístico, y - como una "concesión" al movimiento social- la carrera de Lengua y Cultura Maya.

En estos trabajos se documentan las formas que asume el "multiculturalismo neoliberal" (Hale, 2004) en este campo, donde contradictoriamente la educación superior intercultural puede constituir un riesgo al reconocimiento y la exigibilidad de derechos de los pueblos originarios y servir a formas de cooptación (Llanes, 2008) y de regulación social (Aguilar, 2011). Varios autores coinciden en ello. Hernández y Lemus (2013) y Hernández (2012), por ejemplo, describen un conflicto fundacional en torno a la ubicación de la Universidad Intercultural del Estado de Puebla, que inició funciones en 2006; con testimonios de pobladores, argumentan que la elección de la sede tenía como trasfondo la necesidad política de restar poder a la Organización Independiente Totonaca (OIT), con una trayectoria de más de treinta años de movilización social.

Finalmente, el caso de la Universidad Intercultural de Guerrero es representativo de esta "interculturalidad conflictiva” (Gasché, 2008), pero además introduce en esta revisión las iniciativas de educación superior intercultural que se construyen bajo un perfil autonómico. En la región de La Montaña de Guerrero se inauguró en 2007 la Universidad Intercultural de los Pueblos del Sur (UNISUR), un proyecto que operaba en cinco municipios de la región y que era concebido como "un espacio de educación no formal para jóvenes indígenas, desarrollando un modelo orientado al medio rural y fomentando capacidades en las comunidades y familias, por medio de asistencia técnica, transfiriendo tecnología y conocimientos especializados en actividades agropecuarias" (Guzmán et al. 2014), pero además con un claro componente crítico, decolonial y emancipatorio. La UNISUR se inscribe en un contexto sociohistórico teñido de luchas reivindicativas: la del magisterio educativo regional, la electoral vinculada a la oposición partidaria y la lucha de las organizaciones independientes de la región de La Montaña, que confluyeron en la demanda de una universidad con un perfil propio y políticamente pertinente.

La negociación por el reconocimiento de los planes de estudio y los recursos para operarlo se rompió en 2006 con el sorpresivo anuncio del proyecto de creación de la Universidad Intercultural del Estado de Guerrero (UIEG), adscrita a la CGEIB. Según los actores, esta iniciativa no consideraba las demandas campesinas e indígenas que se habían recogido en el planteamiento de la UNISUR y no se había seguido proceso de consulta alguno (Alonso et al., 2014). Polarizadas las posiciones en una coyuntura de fuerte tensión, finalmente ambas instituciones abrieron sus puertas al año siguiente y en el mismo municipio; la UNISUR, sin reconocimiento oficial de estudios, contó con el apoyo de las comunidades de La Montaña y de 
redes académicas y civiles a nivel nacional ${ }^{8}$ que a la fecha están activas.

López Rangel (2009 y s.f.) documentó etnográficamente este proceso, enfatizando las pugnas políticas que llevaron a que la iniciativa se contrapunteara con la propuesta oficial, en el contexto de la micropolítica regional y de la política educativa intercultural del Gobierno federal. La autora señala que este marco conflictivo se convirtió en un recurso discursivo útil para la definición misma de la UNISUR como expresión de la lucha étnica por el reconocimiento de los derechos educativos indígenas. Esta primera tensión inscribe el proyecto en la arena política de los procesos hegemónicos y contrahegemónicos, que explican la complejidad de las relaciones contemporáneas entre la etnicidad política y las políticas educativas.

Diversos trabajos de divulgación reportan proyectos de educación superior intercultural que - como la UNISUR - se mueven en el terreno de las políticas instituyentes de los pueblos ${ }^{9}$ (Castoriadis, 1997). Oaxaca es semillero de iniciativas con base social comunitaria y un enfoque intercultural; sin embargo, hay una tendencia a distanciarse de esa denominación por su identificación con las políticas gubernamentales neoindigenistas, y porque para muchos actores sociales que son docentes indígenas la educación intercultural desdibuja la lucha social y las reivindicaciones educativas y sociales del magisterio bilingüe oaxaqueño.

En su lugar, la educación comunitaria es una propuesta alterna basada en el discurso filosóficopolítico de la comunalidad. ${ }^{10}$ En su dimensión pedagógica e identitaria, la educación comunitaria se propone formar sujetos identificados con la cultura y las lenguas originarias, y practicantes de los valores de la filosofía comunal colectivista, sea en el contexto de la comunidad rural o de la comunidad ampliada en el espacio urbano. En su dimensión operativa y como proyecto político, se ha negociado como un subsistema escolar diferencial para comunidades indígenas, articulado en todos los niveles educativos - desde preescolar hasta educación superior-y con reconocimiento oficial; se demanda al Estado ser garante del derecho a la educación pública pertinente y de calidad, y proporcionar los recursos materiales y profesionales para la continuidad de la formación (Maldonado, 2011).

En el nivel superior de la educación comunitaria se halla la Licenciatura en Educación Intercultural Comunitaria de la Unidad de Estudios Superiores de Alotepec (UESA-LEIC), que inició funciones en el ciclo escolar 2011-2012 en la región mixe de Oaxaca, y que es reseñada por Maldonado (2014). Originalmente estaba pensada para dar cobertura docente al subsistema de bachilleratos de la educación comunitaria, formando "educadores comunales" (Briseño, 2013); sin embargo, los vínculos de esta iniciativa con representantes del movimiento indígena y con redes académicas amplias, así como el perfil identitario de sus estudiantes, que son jóvenes que provienen de distintas regiones de Oaxaca, fueron imprimiendo a la formación un sentido crítico de las desigualdades y contestatario de las políticas neoindigenistas y neoliberales, así como de sus impactos en la vida comunitaria. En 2015, en una coyuntura de falta de reconocimiento oficial a sus estudios, los estudiantes incrementaron su participación política en la licenciatura y conformaron el Movimiento UESA, que recientemente logró el reconocimiento de su plan de estudios. Institucionalmente, la UESA-LEIC se mantiene como un proyecto desarrollado por el Colegio Superior para la Educación Integral Intercultural de Oaxaca (CSEIIO), en coordinación con autoridades municipales de Santa María Alotepec, Mixe, y una red amplia de apoyos a nivel gubernamental, social y académico.

La certificación de los estudios profesionales de estas iniciativas instituyentes es la punta de una problemática profunda que involucra la gestión escolar como un mecanismo de control y un terreno de lucha. Los proyectos educativos gestados fuera del sistema de las universidades interculturales han de ajustarse a los lineamientos oficiales nacionales de cada subsistema, y a sus mecanismos administrativos y de gestión, para validar legalmente los estudios. El reconocimiento de derechos suele ser letra muerta en la esfera de la gestión escolar, ya que la estructura burocrática administrativa, la rigidez de la normatividad y la inexistencia de márgenes reales de decisión autónoma en la gestión, son 
prácticas que siguen restringiendo el reconocimiento de derechos educativos y culturales, limitando la diversificación curricular y la interculturalización de las prácticas universitarias.

Esto fue lo que desgastó, por ejemplo, el proyecto de la Universidad Indígena Latinoamérica en Tabasco (UIL), a la que se le negó la validación durante dos periodos gubernamentales, y con ello se vio forzada a cerrar. Pocos meses después, según las crónicas periodísticas, ${ }^{\text {Il }}$ se inauguró la Universidad Intercultural del Estado de Tabasco (UIET), y en marzo de 2015 el gobernador anunció la reapertura de la UIL, ahora como una sede de la UIET.

Otro caso, en el mismo sentido, es el del Centro Universitario Regional del Totonacapan, Veracruz (CURT) abierto en el año 2000 y documentado por Cantú et al. (2006) -quien entrevistó a un actor involucrado en el proceso-. El CURT también se enfrentó a estos mecanismos de control al no aprobarse su mapa curricular original, que incluía formación etnopolítica; optaron entonces por buscar alternativas periféricas y necesariamente limitadas, como talleres extracurriculares, para plasmar la dimensión identitaria del proyecto. Esta problemática ha sido reportada en estados de conocimiento previos y sigue siendo señalada.

Guillermo Estrada (2008) hace un recuento de la experiencia del Centro de Estudios Ayuuk-Instituto Superior Intercultural Ayuuk (CEA-ISIA), ubicado en Jaltepec de Candayoc, en la región mixe baja de Oaxaca, que se configura como un contexto multiétnico. Inició funciones en 2006 como una iniciativa negociada por la organización étnica Servicios del Pueblo Mixe (SER) con el gobierno del estado y el Sistema Universitario Jesuita (SIJ), gracias a liderazgos étnicos importantes. El ISIA se define como intercultural, ${ }^{12}$ no tiene un perfil étnico-comunitario, aunque en el proceso previo a su apertura el equipo promotor, formado tanto por activistas mixes como por actores académicos y religiosos, desarrolló tareas de investigación cultural para elaborar el currículo y diseñar el modelo educativo. Asimismo, promovió la colaboración con las autoridades comunales, quienes donaron el terreno y apoyan con trabajo colectivo. Ampliar la participación comunitaria en el proyecto es fundamental ya que se pretende que llegue a ser autogestivo (Estrada et al., 2006).

Finalmente, aunque no se hallan documentadas, existen otras iniciativas de este corte. En Santa María Tlahuitoltepec, en la región mixe alta, una larga trayectoria de gestión de proyectos educativos comunitarios fundados en la vitalización identitaria, el arraigo territorial y el desarrollo del conocimiento, la lengua y las prácticas culturales ayuuk (González Apodaca, 2008), gestó en años recientes un proyecto de educación superior, la Universidad Comunal Intercultural del Cempoaltépetl (UNICEM). ${ }^{13}$ Esta universidad instituyente, con un perfil comunitario y etnogenético, es una iniciativa de jóvenes profesionistas mixes recomunalizados, avalada por las autoridades municipales. Se propone como espacio de formación de nuevos cuadros intelectuales y profesionales con perspectiva comunitaria y competencias amplias para intervenir propositivamente en los problemas locales. La UNICEM se sostiene por colaboraciones financieras y en especie que se gestionan a través de sus redes académicas multiculturales, ${ }^{14}$ ya que no cuenta con el reconocimiento del Instituto Estatal de Educación ni se sustenta con fondos públicos. Si bien esto supone, hipotéticamente, que la matrícula sea muy reducida, el reconocimiento oficial de estudios no ha sido hasta ahora una prioridad del equipo profesionista-docente, que es originario de la comunidad.

\section{Conclusiones}

Para concluir esta revisión, interesa señalar algunos retos que se desprenden de los trabajos y de su contraste con los pronunciamientos hechos por actores del campo de la educación intercultural en México, recogidos en el documento "Diálogo entre academia, organizaciones comunitarias de base, organizaciones de docentes y de la sociedad civil y hacedores de políticas públicas en educación indígena", que retomamos aquí. ${ }^{15}$

La educación superior intercultural en México se caracteriza por su diversidad y por la desigualdad de condiciones institucionales en que se desarrollan las iniciativas y sus actores. Aun atendiendo 
únicamente a su constitución inicial, sus entramados actorales y la semántica de "lo intercultural" como su signo, se perfila claramente como un conjunto heterogéneo, dinámico y con posiciones diferenciales en términos de reconocimiento, recursos y márgenes reales de autonomía de las iniciativas instituidas e instituyentes. El campo aparece como un continuum diverso y multidireccional, aun en el mismo sistema de universidades interculturales oficiales.

Las características particulares de cada centro educativo se construyen con la intervención de tres variables que se intersectan: a) los contextos sociopolíticos regionales en los que se ubican, b) el nivel de participación, negociación e intereses entre los diferentes segmentos de actores involucrados y c) los sentidos que se dan a la educación intercultural.

En los casos documentados sobresale la tensión entre la interculturalidad entendida como la expresión de un conflicto histórico de poder diferencial, que para ciertos actores requiere ser explicitado, y su concepción como horizonte futuro fundado en la promoción de valores éticos o circunscrito a la exaltación positiva de la diferencia cultural; se perfila también la influencia del pensamiento intercultural latinoamericano, donde este carácter trasciende la reivindicación de la diferencia cultural en sí misma para visibilizar los mecanismos y las estructuras de desigualdad, y denunciar críticamente los enfoques de "interculturalidad funcional" a las políticas neoliberales.

El reto para las instituciones de educación superior consiste en dejar de ser meramente una política pública que propone una "acción afirmativa" que compensa la falta de acceso a las universidades convencionales, "reafirmativa" en cuanto pretende valorar y fortalecer la identidad y los conocimientos culturales en los estudiantes y "reiterativa" ya que, al no tomar en cuenta el tema de la desigualdad, reproduce patrones de relaciones de género, de clase e interétnicas. Esto implica trascender hasta convertirse en instituciones impulsoras de cambios estructurales, lo que se encuentra en relación con las demandas indígenas y de otras organizaciones sociales que laboran en el contexto donde se insertan estas instituciones.
Lo anterior indica la importancia de ampliar la participación de estos actores no sólo a nivel consultivo, sino de diálogo y negociación real con académicos, agentes de gobierno e incluso organismos multilaterales, tanto en su génesis como en su desarrollo. Sobre esto tienen mucho que decir aquellas iniciativas que se gestan fuera del aparato oficial de la CGEIB, que hacen efectivo el derecho que les otorgan algunas leyes nacionales y convenios internacionales para demandar una ciudadanía étnica (De la Peña, 1995) en la que los actores indígenas puedan decidir sobre el tipo de educación que quieren para sus jóvenes.

Con todo, estas investigaciones en México parecen conformar todavía un campo emergente fuertemente orientado hacia lo instituido bajo el manto rector de la política intercultural oficial, en detrimento de investigaciones que profundicen en la agencia de los actores y en las tramas socioculturales y políticas de las iniciativas. Se presenta el reto de ampliar y profundizar este debate desde las experiencias concretas y sus particulares contextos sociohistóricos, lo que supone asumir que la educación superior intercultural desde los pueblos indígenas y afrodescendientes, así como el enfoque de interculturalidad para todos, requieren de un componente político para moverse selectivamente desde "lo propio" hasta "lo otro".

Lo anterior es evidente ante las brechas que se reportan entre los responsables de la política educativa, los operadores de las acciones, las financiadoras multilaterales y recientemente privadas, los académicos ylasiniciativas dela sociedad civily de las organizaciones de base, partícipes de la educación superior intercultural. La inercia de las burocracias administrativas imposibilita un diálogo efectivo, y la relación de las iniciativas con el Estado, así como la definición del tipo de vinculación deseado, siguen mostrando la tensión entre la institucionalización de los proyectos, su financiamiento público y su reconocimiento oficial, por un lado, y el rango de autonomía y margen de decisión por otro. Estos obstáculos no son nuevos; han sido señalados reiteradamente por las investigaciones y en foros de debate e intercambio sobre la educación intercultural en México. 
De las dinámicas interactorales registradas, se reitera la necesidad y el reto de "impulsar una concepción amplia de participación social orientada a la búsqueda de una mayor horizontalidad de las instituciones, normas y políticas educativas que operan en contextos indígenas e interculturales, como condición para avanzar hacia la equidad y la pertinencia de las iniciativas" (González Apodaca, 2010: 12), y esta participación:

\section{[...] abarca diferentes ámbitos interactorales: a) el} desarrollo del proyecto en sí, con los espacios, redes y actores sociales implicados; b) la conducción y toma de decisiones institucionales; c) la legitimidad de la iniciativa y del conocimiento que genera; d) la esfera del financiamiento y del reconocimiento, y e) la relación con las instituciones del estado (González Apodaca, 2010: 13).

Asimismo, considerando las tensiones entre el marco participativo y contextualizado del cual surgen las iniciativas instituyentes de educación superior intercultural y sus posibilidades de desarrollarse y de impactar a nivel de política pública, sigue siendo imperativo identificar elementos comunes que deban ser fortalecidos desde las instituciones y la normatividad educativas, así como considerar el establecimiento de políticas, programas y acciones diferenciados y focalizados, inclusivos, pertinentes, flexibles y continuos, mas no de modelos replicables, prescriptivos y estandarizados (González Apodaca, 2010: 14).

Finalmente, la dimensión política de la educación superior intercultural supone encarar nuevos retos en los contextos contemporáneos, entre ellos asumir preguntas urgentes relacionadas con los posicionamientos y sentidos político-pedagógicos de la formación universitaria intercultural, así como de la investigación en educación intercultural y sus compromisos ético-políticos, en escenarios de creciente precarización, migración, asalarización y violencia, políticas neoliberales predatorias y estructuras múltiples de desigualdad.

\section{Notas}

${ }^{1}$ El principal nicho de producción es la Universidad Veracruzana Intercultural(UVI), donde sus estudiantes y académicos realizan estudios sobre las universidades interculturales en general y sobre su caso particular.

${ }^{2}$ Las doce instituciones pertenecientes a la CGEIB son: la Universidad Intercultural del Estado de México - la primera que surgió en el año 2003-, la Universidad Intercultural del Estado de Tabasco, la Universidad Intercultural de Chiapas, la Universidad Intercultural del Estado de Puebla, la Universidad Intercultural del Estado de Guerrero, la Universidad Intercultural Maya deQuintana Roo,la Universidad InterculturaldelEstado de Nayarit, la Universidad Intercultural del Estado de Hidalgo, la Universidad Intercultural Indígena de Michoacán y la Universidad Veracruzana Intercultural, que se desprende de la Universidad Veracruzana. Además, están la Universidad Intercultural del Estado de San Luis Potosí y la Universidad Autónoma Indígena de México, cuyos orígenes fueron independientes y después fueron adscritas a esta estructura.

${ }^{3}$ De hecho, sus antecedentes se vinculan con la formación de actores indígenas como intermediarios entre las comunidades y el Estado. Uno de los primeros intentos de profesionalización indígena es la creación de la Licenciatura en Etnolingüística del Centro de Investigaciones y Estudios Superiores en Antropología Social (entonces CIS-INAH), en 1979, orientada a profesionalizar a promotores culturales que trabajaban en diversas regiones del país (Martínez Casas, 2011). En el ámbito de la formación docente, en los años ochenta se creó la Licenciatura en Educación Primaria para el Medio Indígena (LEPMI) de la Universidad Pedagógica Nacional.

${ }^{4}$ Por su amplitud, en este artículo no está considerado este ámbito, cuyo carácter interactoral y sus marcos conceptuales, epistemológicos y tramas etnopolíticas serán objeto de una revisión posterior. La producción de la última década ha sido reportada por Von Groll, Keyser y Silva (2013).

${ }^{5}$ La propuesta del CESDER no era diferencial para población indígena; sin embargo, incorporó un eje 
intercultural transversal a la formación. Experiencias similares, aunque menos consolidadas, son: el Centro Universitario Regional del Totonacapan, creado en el año 2000 en Papantla, Veracruz, la Universidad Indígena Latinoamericana en Tabasco en 2001, y en 1995 la Universidad de la Montaña en Chiapas, que recientemente cerró sus puertas. Asimismo, destacan la Universidad de los Pueblos del Sur (UNISUR) en el contexto multiétnico de Guerrero, vinculada con el movimiento social de los pueblos de La Montaña y sus iniciativas autonómicas, el Instituto Superior Intercultural Ayuuk en Jaltepec de Candayoc, la Universidad Comunal del Cempoaltéptl (UNICEM) en Santa María Tlahuitoltepec y la Licenciatura en Educación Intercultural Comunitaria de la Unidad de Estudios Superiores de Alotepec (UESA-LEIC), estas tres en comunidades mixes de Oaxaca, con perfiles y redes muy distintas entre sí. Algunas de estas iniciativas se reportan más adelante.

${ }^{6}$ Las perspectivas del estudiantado se recogen en análisis biográficos y estudios de trayectorias que por su número y características metodológicas, se revisan en un trabajo aparte.

7 La Universidad Veracruzana Intercultural, donde el autor reporta que los profesores sí consideran importantes los conocimientos locales.

${ }^{8}$ Instituciones como la Universidad Nacional Autónoma de México, la Universidad Autónoma de la Ciudad de México, el Centro de Investigaciones y Estudios Superiores en Antropología Social y el Instituto Politécnico Nacional. Varios profesores de estas instituciones colaboran con el proyecto UNISUR.

${ }^{9}$ Documentadas ampliamente en otro trabajo (González y Rojas, 2013); únicamente referimos los trabajos más recientes.

10 Acuñado por intelectuales oaxaqueños, zapotecos, mixes y no indígenas, como Jaime Martínez Luna (2010), Floriberto Díaz (Robles y Cardoso, 2007) y Juan José Rendón (2003). En años recientes la propuesta teórica y filosófico-política de la comunalidad ha sido apropiada y rediscutida por jóvenes profesionistas, activistas y académicos vinculados al movimiento social (Aquino, 2013).
${ }^{11}$ Periódico La Verdad del Sureste, 30 de marzo de 2015.

${ }^{12}$ Ofrece las licenciaturas en Comunicación Intercultural, Administración y Desarrollo Sustentable y Educación Intercultural. Disponible en: www.isia.edu.mx/.

${ }^{13}$ Ver: www.unicem.tlahuitoltepec.com

${ }^{14}$ Mantiene vínculos con redes como el CESDER en Puebla, el Programa México: Nación Multicultural de la UNAM, la UNISUR y la UCI-RED, entre otros.

15 Producto del ler Encuentro de experiencias de formación, asesoría, acompañamiento y diseño de materiales didácticos en contextos interculturales y bilingües en México, convocado por el CIESAS y la UNICEF (González Apodaca, 2010).

\section{Referencias bibliográficas}

Aguilar, Mariana del Rocío (2011). "Universidades interculturales, adjetivo o sustantivo. El reto de una educación para y en la diversidad". Ponencia presentada en el XI Congreso Nacional de Investigación Educativa.

Alonso, Lorena, Víctor Hernández y Edgardo Solís (2014). "La universidad intercultural de los pueblos del sur. Una opción de educación no formal para la población indígena en el estado de Guerrero". En RMIE, vol. 19, núm. 66, pp. 103-128.

Aquino, Alejandra (2013). "La comunalidad como epistemología del Sur, aportes y retos", En Cuadernos del Sur, vol. 18, núm. 34, enero-junio, pp. 7-20.

Bailleres, Diana (2010). "Reflexiones sobre la experiencia de la educación intercultural en el Estado de México". En Eduardo Sandoval, Ernesto Guerra y Ricardo Contreras (comp.), Políticas públicas de educación superior intercultural y experiencias de diseños educativos. España: Universidad de Málaga. Disponible en: http://www. eumed.net/libros/2010e/830/index.htm (consultado el 20 de noviembre 2014).

Bello, Juan (2011). "De la educación bilingüe bicultural, a las universidades interculturales en México". En Eduardo Sandoval, Ernesto Guerra y Ricardo Contreras (comp.), Políticas públicas de educación superior intercultural y experiencias de diseños educativos. España: Universidad de Málaga. Disponible en: http://www. 
eumed.net/libros/2010e/830/index.htm (consultado 20 de noviembre 2014).

Bertely, María (coord.) (2003). "Educación, derechos sociales y equidad. La investigación educativa en México 1992-2002", t. I, Educación y diversidad cultural. México: Consejo Mexicano de Investigación Educativa, pp. 85-102.

Bertely, María (2007). Conflicto intercultural, educación y democracia activa en México: ciudadanía y derechos indígenas en el movimiento pedagógico intercultural bilingüe en Los Altos, la Región Norte y la Selva Lacandona de Chiapas. México: CIESAS.

Bertely, María (2011). "Educación superior intercultural". En Perfiles Educativos, vol. 33, enero, pp.66-77.

Bertely, María (2013). "Debates conceptuales sobre educación multicultural e intercultural". En María Bertely, Gunther Dietz, María Guadalupe Díaz Tepepa (coord.) Multiculturalismo y educación 2002-2001. México: COMIE, ANUIES. México, pp. 41-80.

Bertely, María y Erica González Apodaca (2003). Educación y diversidad cultural, t. I. México: COMIE.

Bertely, María, Gunther Dietz y María Guadalupe Díaz (2013). Multiculturalismo y educación. México: COMIE, ANUIES.

Briseño, Julieta (2013). "La comunalidad como epistemología del Sur, aportes y retos". En Cuadernos del Sur, vol. 18, núm. 34, enero-junio, pp. 29-37.

Cantú, Mónica, Alejandro Delgado, José Lara, Rebeca de los Santos y Jorge Ramírez (2006). "Centro Universitario Regional del Totonacapan (CURT)". En Experiencias Innovadoras en Educación Intercultural, vol. 2, pp. 141-154. México: CGEIB.

Castoriadis, Cornelius (1997). "El imaginario social instituyente". En Zona Erógena, núm. 35.

De la Peña, Guillermo (1995). "La ciudadanía étnica y la construcción de 'los indios' en el México contemporáneo". En Revista Internacional de Filosofía Política, núm. 6, pp. 116-140.

Dietz, Gunther (2008). "La experiencia de la Universidad Veracruzana Intercultural”. En Daniel Mato (coord.), Diversidad cultural e interculturalidad en educación superior. Experiencias en América Latina. Caracas: IESALC, UNESCO, pp. 359-370.\}
Dietz, Gunther (2009). "Los actores indígenas ante la 'interculturalización' de la educación superior en México: iempoderamiento o neoindigenismo?" En Revista Latinoamericana de Educación Inclusiva, vol. 3, núm. 2, pp. 55-75.

Dietz, Gunther (2014). "Diversidad e interculturalidad en la Universidad: logros y desafíos desde el contexto mexicano". En Juan Ansion y Ana María Villacorta (coords.), Qawastin ruwastin. Viviendo y haciendo. Encuentros entre sujetos del conocimiento en la universidad. Lima: Pontificia Universidad Católica de Perú, RIDEI, PUCP, pp. 71-91.

Dietz, Gunther y Laura Mateos (2011). Interculturalidad y educación intercultural en México. México: SEP-CGEIB.

Estrada, Guillermo (2006). Hacia dónde vamos. Un diagnóstico de la región Mixe. Oaxaca: CEA-UIIA.

Estrada, Guillermo (2008). "Sobre la experiencia de Centro de Estudios Ayuuk-Universidad Indígena Intercultural Ayuuk". En Daniel Mato (coord.), Diversidad cultural e interculturalidad en educación superior. Experiencias en América Latina. Caracas: IESALC, UNESCO, pp. 371-382.

Fábregas, Andrés (2008). "La experiencia de la Universidad Intercultural de Chiapas". En Daniel Mato (coord.), Diversidad cultural e interculturalidad en educación superior. Experiencias en América Latina. Caracas: IESALC, UNESCO, pp. 339-348.

Fábregas, Andrés (2009). “Cuatro años deeducación superior intercultural en Chiapas, México". En Daniel Mato (coord.), Instituciones interculturales de educación superior en América Latina. Procesos de construcción, logros, innovaciones y desafios. Caracas: UNESCO, IESALC, pp. 25l-278.

Flores, José Joaquín (2009). "Las luchas indias y sus intelectuales". En Veredas, núm. 18, pp. 193-213. México: UAM-Xochimilco.

Gasché, Jorge (2008). "La motivación política de la educación intercultural indígena y sus exigencias pedagógicas. ¿Hasta dónde abarca la interculturalidad?" En María Bertely, Jorge Gasché y R. Podestá, Educando en la diversidad. Quito, Ecuador: Ediciones Abya-Yala.

González Apodaca, Erica E. (2008). Los profesionistas indios en la educación intercultural. Etnicidad, 
intermediación y escuela en territorio mixe. México: Universidad Autónoma Metropolitana, Editorial Juan Pablos.

González Apodaca, Erica E. (2009). “Acerca del multiculturalismo, la educación intercultural y los derechos indígenas en las Américas". En CPU-e:Revista de Investigación Educativa, núm. 9, julio-diciembre.

González Apodaca, Erica E. (comp.) (2010). Diálogo entre academia, organizaciones comunitarias de base, organizaciones de docentes y de la sociedad civil y hacedores de políticas públicas en educación indígena, ler Encuentro de experiencias de formación, asesoría, acompañamiento y diseño de materiales didácticos en contextos interculturales y bilingües en México. México: UNICEF, CIESAS.

González, Felipe (2007). "Cultural y desarrollo desde la interculturalidad. Breve recuento de la primera universidad intercultural de México". En RA Ximhai, mayo-agosto, año/vol. 3, núm. 2, pp. 243-272. Sinaloa: Universidad Autónoma Indígena de México

González Apodaca, Erica E. y Angélica Rojas (2013), "Proyectos locales, autonomía educativa y resistencia indígena”. En Bertely, María, Gunther Dietz y María Guadalupe Díaz (coord.), Multiculturalismo y educación. México: COMIE, ANUIES, pp. 383-414.

Guerra, Ernesto (2008). "La experiencia educativa de la UniversidadAutónoma Indígenade México”. EnDaniel Mato (coord.), Diversidad cultural e interculturalidad en Educación Superior. Experiencias en América Latina. Caracas: IESALC, UNESCO, pp. 349-358.

Guerra, Ernesto y María Eugenia Meza (2009). “El corto y sinuoso camino de la Universidad Autónoma Indígena de México (UAIM)". En Daniel Mato (coord.), Instituciones interculturales de educación superior en América Latina. Procesos de construcción, logros, innovaciones y desafíos. Caracas: UNESCO, IESALC, pp. 215, 250.

Guitart, Moisés Esteban y María Rivas (2008). "La propuesta de las universidades interculturales en México frente al pluralismo cultural: el caso de Chiapas". En Documentación Social, núm. 15l. Madrid: Caritas Española Editores, pp. 147-162.

Hale, Charles (2004). "Racismo cultural. Notas desde Guatemala de una paradoja americana”. En Meike Heckt y Gustavo Palma Murga (coord.), Racismo en Guatemala. De lo políticamente correcto a la lucha antirracista. Guatemala: AVANCSO, pp. 21l-234.

Hernández, Sergio (2012). “Experiencias en la generación de un modelo de investigación intercultural en la Universidad Intercultural del Estado de Puebla". Ponencia presentada en el Congreso Desafíos y Horizontes de Cambio: México en el siglo XXI. Ciudad de México, del 26 de febrero al $1^{\circ}$ de marzo de 2012.

Hernández, Sergio y Genaro Lemus (2013). “Vinculación comunitaria en la UIEP: aprendizajes en proceso". Ponencia presentada en el $9^{\circ}$ Congreso Nacional de la Asociación Mexicana de Estudios Rurales. Universidad de Guadalajara, del 5 al 8 de marzo 2013. Lehman, David (2015). "Convergencias y divergencias en la educación superior intercultural en México". En Revista Mexicana de Ciencias Políticas y Sociales, Nueva Época, año LX, núm. 223, enero-abril pp. 133-170. México: UNAM.

Llanez, Genner (2008). "Interculturalización fallida. Desarrollismo, neoindigenismo y universidad intercultural en Yucatán, México". En Trace, núm. 53, junio, pp. 49-63.

López Hurtado, Luis. E. (2010). "Interculturalidad, educación y política en América Latina: perspectivas desde el sur. Pistas para una investigación comprometida y dialogal". En $\chi$ Congreso Nacional de Investigación Educativa. Veracruz, 2009. Conferencias magistrales. Memoria electrónica (CD). México: COMIE. López, Álvaro (2013). "La investigación colectiva como marcoético-político en la construcción de proyectos de "Vida Buena" en la UVI sede Totonacapan". Ponencia presentada en $9^{\circ}$ Congreso Nacional de la Asociación Mexicana de Estudios Rurales. Universidad de Guadalajara, del 5 al 8 de marzo de 2013.

López Rangel, Angélica (2009). Apuestas de educación superior intercultural en la Costa-Montaña de Guerrero. La construcción de la UNISUR desde sus actores y procesos. Tesis de maestría, CIESAS, México.

López Rangel, Angélica (s.f.). Redes de movimientos sociales en la construcción de Otras educaciones interculturales: los unisureños y la REDIIN. Tesis de doctorado en Antropología Social, CIESAS D.F. (documento inédito). 
Maldonado Alvarado, Benjamín (2011). Comunidad, comunalidad y colonialismo en Oaxaca. La nueva educación comunitaria y su contexto. México: CSEIIO.

Maldonado, Carlos (2014). Política intercultural, educación comunitaria y lengua originaria. Historias de vida escolar de los jóvenes de la UES-LEMSC. Tesis de licenciatura, UABJO, Oaxaca.

Martínez Casas, Regina (2011). "La formación de los profesores bilingües indígenas en el México contemporáneo". En Perfiles Educativos, vol. XXXIII, núm. especial, pp. 250-261.

Martínez Luna, Jaime (2010). Eso que llaman comunalidad. Oaxaca, México: Consejo Nacional para la Cultura y las Artes.

Mateos, Laura, Rosa Guadalupe Mendoza y Gunther Dietz (2013). "Diversidad e interculturalidad en la educación superior convencional". En María Bertely, Gunther Dietz y María Guadalupe Díaz (coord.), Multiculturalismo y educación. México: COMIE, ANUIES, pp. 307-308.

Mateos, Laura y Gunther Dietz (2013). "Universidades Interculturales en México". En María Bertely, Gunther Dietz y María Guadalupe Díaz (coord.), Multiculturalismo y educación. México: COMIEANUIES, pp. 349-382.

Mato, Daniel (2008). "Programa regional". En Daniel Mato, Diversidad cultural e interculturalidad en Educación Superior. Experiencias en América Latina. Caracas: IESALC, UNESCO, pp. 21-82.

Medina, Patricia y Bruno Baronnet (2013). "Movimientos decoloniales en América Latina: un abalance necesario desde las pedagogías interculturales emergentes en México. Autonomía, territorio y educación propia”. En María Bertely, Gunther Dietz y María Guadalupe Díaz (coord.), Multiculturalismo y educación. México: COMIE, ANUIES, pp. 415-448.

Navarro, Sergio y Victoria Rojas (2014). "La interculturalidad desde el contexto comunitario. Análisis de la experiencia de la Universidad Intercultural del Estado de Tabasco". En Ezequiel Hernández, Ricardo Contreras y Rubén Ramírez (coord.), Estudios de los procesos interculturales: comunidad, redes, construcciones mediáticas, experiencias organizativas, procesos de construcción y humanismo. México: EUMED, Universidad de Guanajuato.

Ortelli, Paola y Stefano Claudio Sartorello (2011). "Jóvenes universitarios y conflicto intercultural: Estudiantes indígenas y mestizos en San Cristóbal de Las Casas, Chiapas". En Perfiles Educativos, vol. 33, núm. especial, pp. 115-128. Disponible en: http://www.scielo. org.mx/scielo.php?script=sci_arttext\&pid=S018526982011000500011\&elng=es\&tlng=en (consultado el 6 de mayo de 2015).

Padilla, Alberto (2008). "Las universidades indígenas en México. Inclusión o exclusión”. En Veredas, núm. especial, pp.35-59.

Pérez Ruiz, Maya Lorena (2009). “iDe qué hablamos cuando nos referimos a lo intercultural? Reflexiones sobre su origen, contenidos, aportaciones y limitaciones". En Laura Valladares, Maya Lorena Pérez Ruiz y Margarita Zárate Vidal (coord.) Estados plurales. El reto de la diversidad y la diferencia. México: UAM-Iztapalapa, pp. 199-228.

Rendón, Juan José (2003). La comunalidad. Modo de vida en los pueblos indios, t. I. México: Dirección General de Culturas Populares e Indígenas, CONACULTA.

Robles, Sofía y Rafael Cardoso (comp.) (2007). Floriberto Díaz. Escrito. Comunidad: energía viva del pensamiento mixe, México Nación Multicultural. México: UNAM.

Rockwell, Elsie (2009). La experiencia etnográfica: historia y cultura en los procesos educativos. Buenos Aires: Paidós.

Sandoval, Eduardo y Ernesto Guerra (2007). "La interculturalidad en la educación superior en México". En Ra Ximhai, mayo-agosto, vol. 3, núm. 2, pp. 273-288.

Sandoval, Eduardo y María Eugenia Meza (2010). "Las dimensiones de la interculturalidad: el discurso de los rectores de las universidades interculturales". En Eduardo Sandoval, Ernesto Guerra y Ricardo Contreras (coord.), Políticas públicas de educación superior intercultural y experiencias de diseños educativos. España: Universidad de Málaga, pp. 36-59. Disponible en: http://www.eumed.net/libros/2010e/830/index.htm (consultado el 20 de noviembre de 2014).

Sartorello, Stefano. C. (2009). "Una perspectiva crítica sobre interculturalidad y educación intercultural bilingüe: el caso de la Unión de Maestros de la Nueva 
Educación para México (UNEM) y educadores independientes en Chiapas". En RevistaLatinoamericana de Educación Inclusiva, vol. 3, núm. 2, pp. 77-90.

Silva, Salvador (2009). "Universidad Comunitaria de San Luis Potosí". En Daniel Mato (coord.), Educación superior, colaboración intercultural y desarrollo sostenible/ buen vivir. Experiencias en América Latina. Caracas: UNESCO, IESAL, pp. 163-182.

UNESCO (1998). Declaración Mundial sobre Educación Superior en el Siglo XXI: Visión y Acción. S.1.: UNESCO.

Velasco, Saúl y Aleksandra Jablonska (2013). "Políticas en educación indígena intercultural”. En María Bertely, Gunther Dietz y María Guadalupe Díaz (coord.), Multiculturalismo y educación. México: COMIE, ANUIES, pp. 117-150.

Vivar, Karla (2014). Trayectoria y desplazamiento de modelos teóricos e ideológicos: de la educación indígena en México. Una crítica antropológica. Tesis de doctorado en Ciencias Sociales, CIESAS Occidente, México.

Von Groll, Maren, Ulrike Keyser y Elías Silva (2013). "Formación de maestros indígenas y no indígenas para la educación indígena e intercultural". En María Bertely, Gunther Dietz y María Guadalupe Díaz (coord.), Multiculturalismo y educación. México: COMIE, ANUIES, pp. 151-184.

Walsh, Catherine (1998). "Interculturalidad en la nueva onda de lo pluri: significados y políticas conflictivas". Ponencia presentada en el III Congreso Latinoamericano de Educación Intercultural Bilingüe, Quito.

Walsh, Catherine (2010). "Interculturalidad crítica y educación intercultural”. En Jorge Viaña, Luis Tapia y Catherine Walsh, Construyendo interculturalidad crítica. La Paz: Convenio Andrés Bello, pp. 75-96. 